Supporting Information

\title{
Touchless Sensing Interface Based on the Magneto-Piezoresistive Effect of Magnetic Microstructures with Stacked Conductive Coating
}

Weiguan Zhang, ${ }^{1,2 \dagger}$ Qinhua Guo, ${ }^{1 \dagger}$ Yu Duan, ${ }^{1}$ Qunhui Xu, ${ }^{1}$ Chao Shang, ${ }^{1}$ Ning $\mathrm{Li},{ }^{2}$ and Zhengchun Peng $^{1 *}$

${ }^{1}$ Center for Stretchable Electronics and Nano Sensors, College of Physics and Optoelectronic Engineering, Shenzhen University, Shenzhen 518060, China

${ }^{2}$ College of Mechatronics and Control Engineering, Shenzhen University, Shenzhen 518060, China

*E-mail: zcpeng@szu.edu.cn

$\dagger$ W. G. Zhang and Q. H. Guo contributed equally to this work. 


\section{Section 1. Derivation of the attractive magnetic force}

\section{Ferromagnetic substrate}
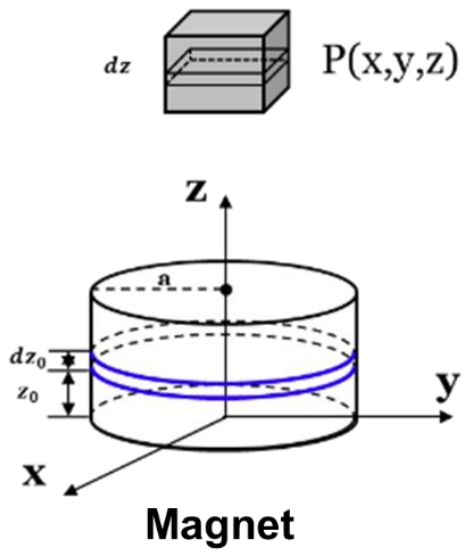

As depicted in the figure, the magnetic proximity sensing model is analyzed in the cartesian coordinate. A cylindrical permanent magnet with radius $a$ and thickness $h$ is considered to be fully magnetized in the z-axis direction. According to the molecular current model, the static magnetic field induced by the magnet in the space out of magnet is equivalently generated by all the molecular currents in the magnet. ${ }^{49}$ Considering the mutual canncellation of adjacent molecular current in uniform magnetized magnet, the superposition of all molecular currents can be viewed as surface current loop only with the strength of $I$, which contributes the magnetic field in the space out of magnet.

Bottom center of the cylindrical magnet is defined as zero points $(0,0,0)$, and a arbitrary point $A$ in magnet is denoted as $\left(x_{0}, y_{0}, z_{0}\right)$. According to the above-mentioned theory, a thin cylindrical layer of magnet between the plane $z_{0}$ and $z_{0}+d z_{0}$ can be equivalently regarded as the surface current loop of $J d z_{0}$, where current density can be calculated by $J=I / h$.

The magnetic field at the point $P(x, y, z)$ induced by the magnet can be expressed as:

$$
\overrightarrow{\boldsymbol{B}}=B_{x} \overrightarrow{\boldsymbol{\imath}}+B_{y} \overrightarrow{\boldsymbol{\jmath}}+B_{z} \overrightarrow{\boldsymbol{k}}=\int_{0}^{h} d B_{x} \overrightarrow{\boldsymbol{\imath}}+d B_{y} \overrightarrow{\boldsymbol{\jmath}}+d B_{z} \overrightarrow{\boldsymbol{k}}
$$

where $d B_{x}, d B_{y}, d B_{z}$ are magnetic induction components at point $P$ in the direction of $x, y, z$ contributed from surface current loop.

According to the Biot-Savart's law, the magnetic induction in the space produced by current element $I$ is expressed as:

$$
d \overrightarrow{\boldsymbol{B}}=\frac{\mu_{0}}{4 \pi} \frac{I d \overrightarrow{\boldsymbol{l}} \times \overrightarrow{\boldsymbol{D}}}{D^{3}}
$$

where $\mu_{0}$ is the magnetic permeability of air $\left(4 \pi \times 10^{-7}\right), \vec{D}$ is a vector from zero points to $P$. 
Concerning the magnet in this work is much larger than the sensor, only the $z$ component of magnetic induction $P$ point is considered in the further calculation.

The transformation between cartesian coordinate and cylindrical coordinate are:

$$
\begin{gathered}
\overrightarrow{\boldsymbol{r}}=(a \cos \theta, a \sin \theta, 0) \\
d \overrightarrow{\boldsymbol{l}}=d \overrightarrow{\boldsymbol{r}}=(-a \sin \theta d \theta, a \cos \theta d \theta, 0) \\
\overrightarrow{\boldsymbol{D}}=\left(-x_{0},-y_{0}, z-z_{0}\right)=\left(-a \cos \theta,-a \sin \theta, z-z_{0}\right)
\end{gathered}
$$

Thus, the magnetic induction can be re-written as:

$$
\begin{aligned}
d B_{z} & =\int_{0}^{2 \pi} \frac{\mu_{0} I\left[a^{2}(\sin \theta)^{2}+a^{2}(\cos \theta)^{2}\right]}{4 \pi\left[(a \cos \theta)^{2}+(a \sin \theta)^{2}+\left(z-z_{0}\right)^{2}\right]^{\frac{3}{2}}} d \theta=\frac{\mu_{0} a^{2} J d z_{0}}{4 \pi\left[a^{2}+\left(z-z_{0}\right)^{2}\right]^{\frac{3}{2}}} \int_{0}^{2 \pi} 1 d \theta \\
& =\frac{\mu_{0} a^{2} J}{2\left[a^{2}+\left(z-z_{0}\right)^{2}\right]^{3 / 2}} d z_{0}
\end{aligned}
$$

And, the $\mathrm{z}$-axis magnetic induction component produced by the magnet at the point $P$ can be calculated as below:

$$
B_{z}(z)=\int_{0}^{h} \frac{\mu_{0} a^{2} J}{2\left[a^{2}+\left(z-z_{0}\right)^{2}\right]^{3 / 2}} d z_{0}=\frac{\mu_{0} J}{2}\left[\frac{h-z}{\sqrt{a^{2}+(h-z)^{2}}}+\frac{z}{\sqrt{a^{2}+z^{2}}}\right]
$$

To simplify the derivation, we assume the $\mathrm{Fe}_{3} \mathrm{O}_{4}$ nanoparticles are uniformly dispersed in the substrate which is with the thickness of $H$ and cross-section area of $S$, and also, a thin layer of ferromagnetic substrate (thickness of $d z$ ) is uniformly magnetized under the magnetic field $B_{z}(z)$. Hence, the magnetic potential energy of the ferromagnetic thin layer can be expressed as below: ${ }^{51}$

$$
d U=\frac{B_{z}(z) d m(z)}{2}=\frac{k B_{z}(z) M(z) d V}{2}=\frac{k B_{z}{ }^{2}(z) \chi d V}{2 \mu_{0}}=\frac{k B_{z}{ }^{2}(z) \chi S}{2 \mu_{0}} d z
$$

where $m(z)$ is the magnetic torque of the ferromagnetic thin layer, $M(z)$ is the magnetization of ferromagnetic thin layer, $k$ is a coefficient related to the weight ratio of $\mathrm{Fe}_{3} \mathrm{O}_{4}$ particles mixed in the thin layer, and $\chi$ is the magnetic susceptibility of the ferromagnetic material.

Then, the magnetic potential energy of the ferromagnetic substrate under the magnetic field $B_{z}(z)$ can be further integrated:

$$
U(L)=\int_{L+h}^{L+h+H} \frac{k B_{z}^{2}(z) \chi S}{2 \mu_{0}} d z
$$

where $L$ is the distance from the upper surface of the magnet to the bottom surface of the ferromagnetic substrate. 
Differentiating the energy by distance will obtain the magnetic force that applied on the substrate as derived below:

$$
\begin{aligned}
F_{z}(L)=\frac{\partial U(L)}{\partial L} & =\frac{k \chi S}{2 \mu_{0}}\left[B_{z}{ }^{2}(L+h+H)-B_{z}{ }^{2}(L+h)\right] \\
& =\frac{k \chi S \mu_{0} J^{2}}{8}\left\{[\varphi(L+H+h)-\varphi(L+H)]^{2}-[\varphi(L+h)-\varphi(L)]^{2}\right\}
\end{aligned}
$$

in which

$$
\varphi(x)=\frac{1}{\sqrt{1+\left(\frac{a}{x}\right)^{2}}}
$$




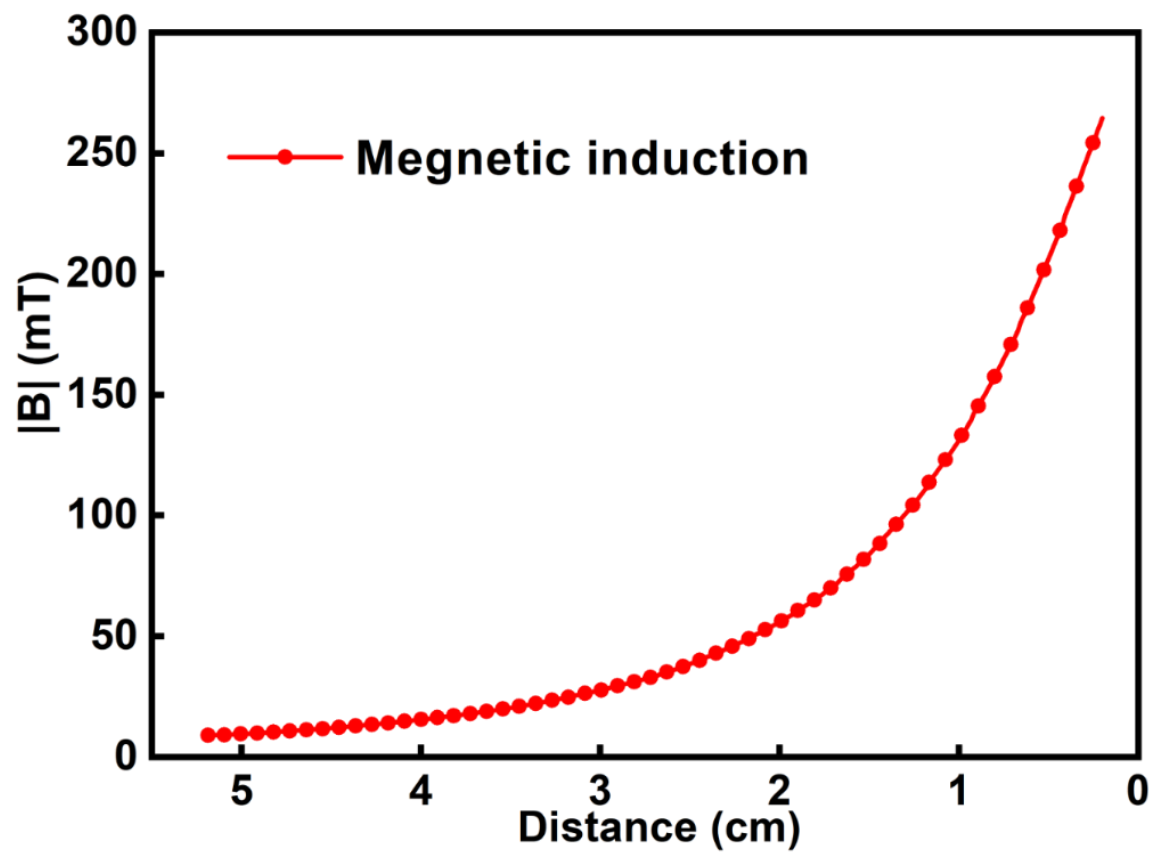

Figure S1. Measured magnetic induction induced by the magnet as a function of the approaching distance. 
(a)

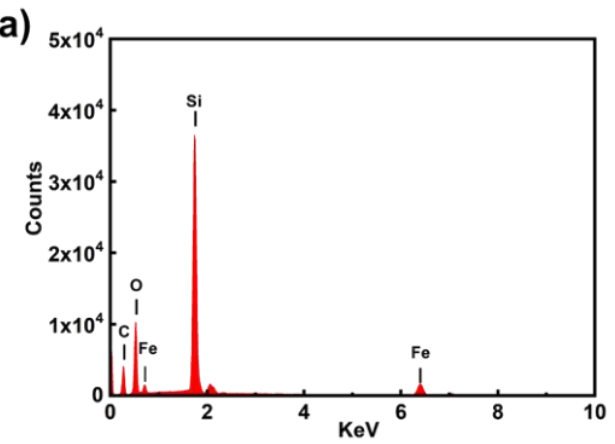

(b)

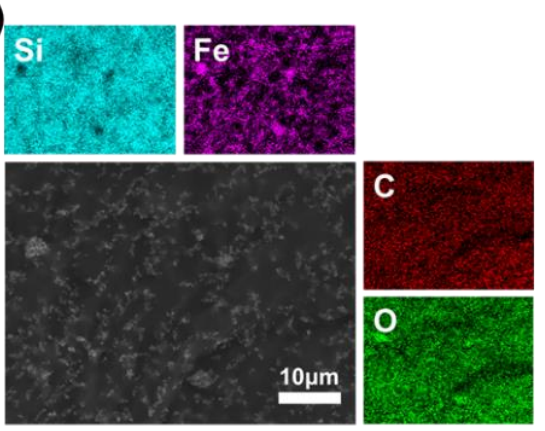

(c)

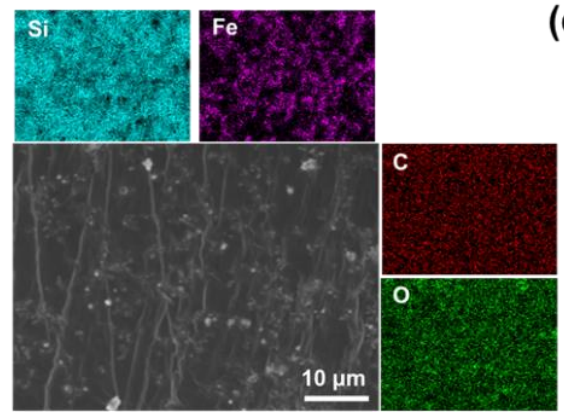

(d)

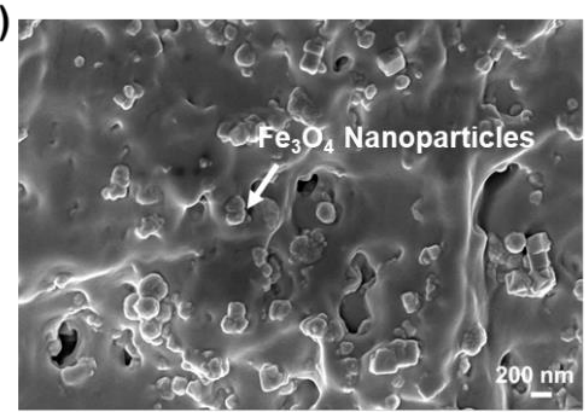

Figure S2. $(a, b)$ EDS analysis and element mapping on the top surface of the MFP substrate.(c) Element mapping on the cross-section of the MFP substrate. (d) View of $\mathrm{Fe}_{3} \mathrm{O}_{4}$ nanoparticles dispersed in the PDMS. 
(a)

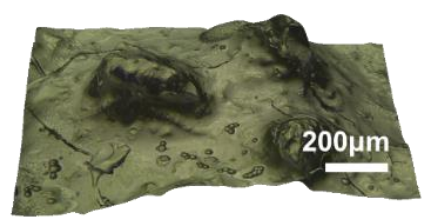

(d)

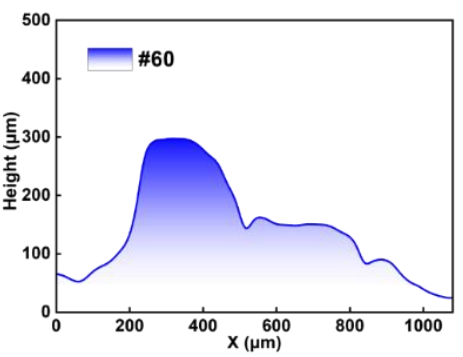

(b)

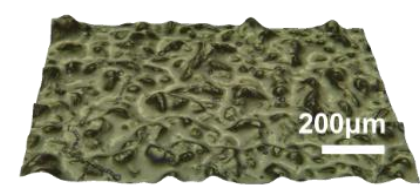

(e)

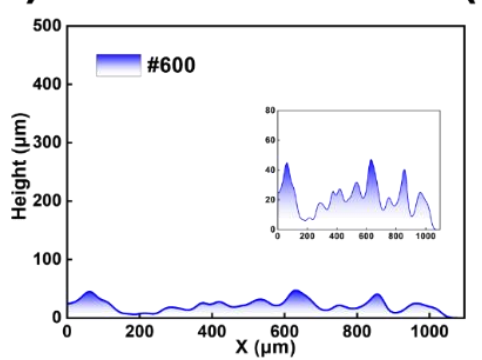

(c)

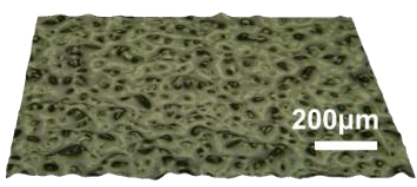

(f)

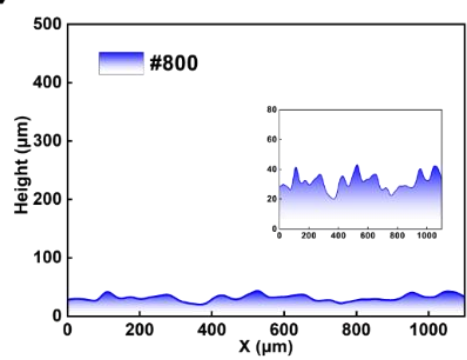

Figure S3. (a-c) 3D microscope images of the MFP substrate demolded from (a) \#60, (b) \#600, and (c) \#800 sandpapers. (d-f) Height profile analysis of the MFP substrate demolded from \#60, \#600, and \#800 sandpapers. 


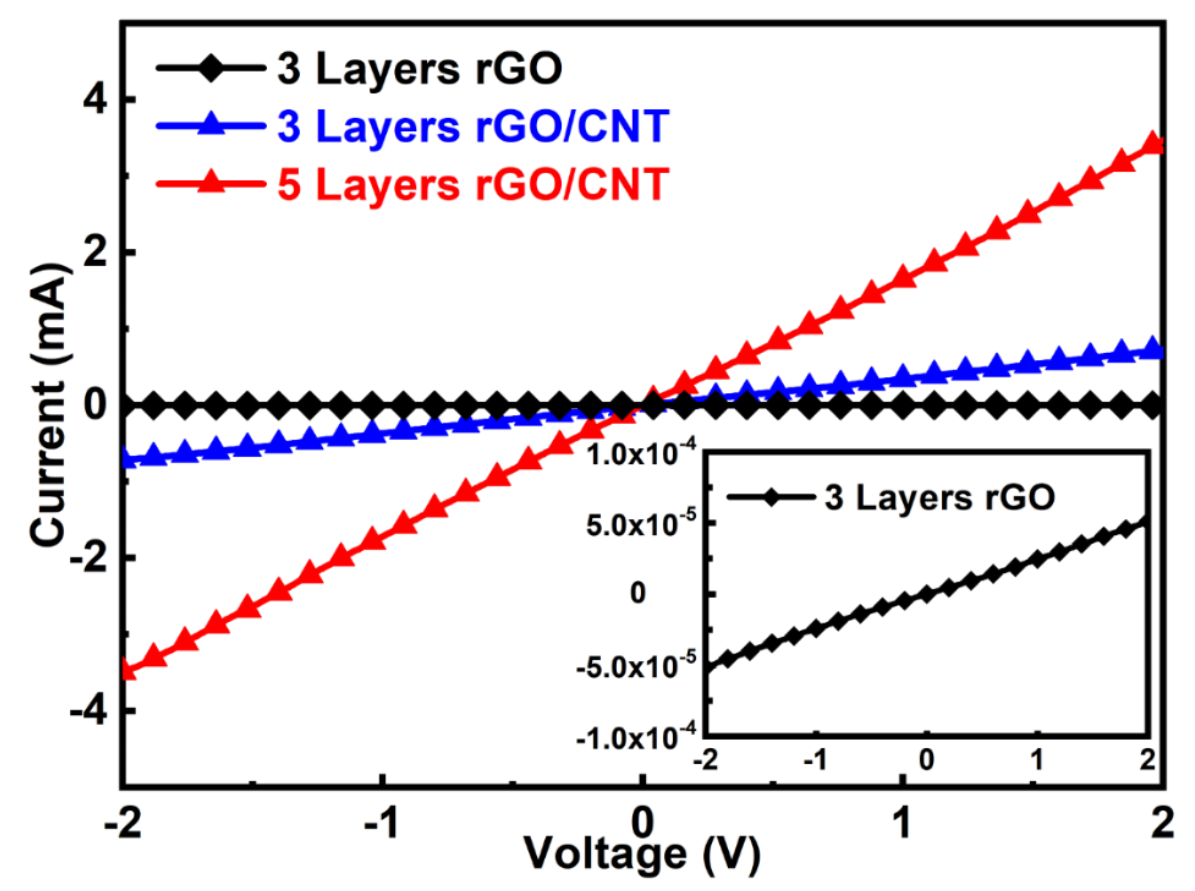

Figure S4. Measured $I-V$ curve of the magneto-piezoresistive proximity sensor with different layer numbers in constructing the 3D stacked piezoresistive network. 


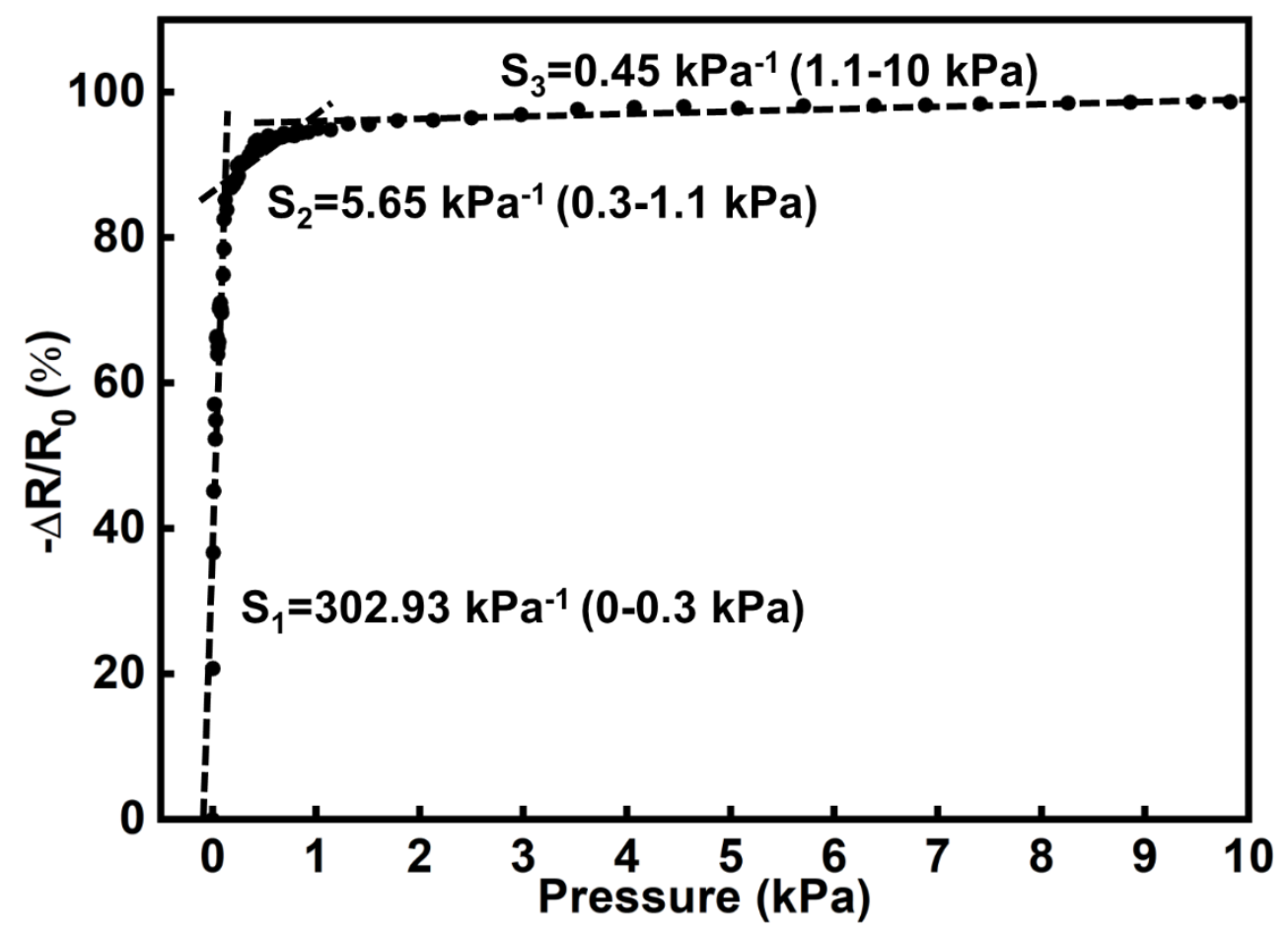

Figure S5. Measured piezoresistive response of the magneto-piezoresistive sensor. 


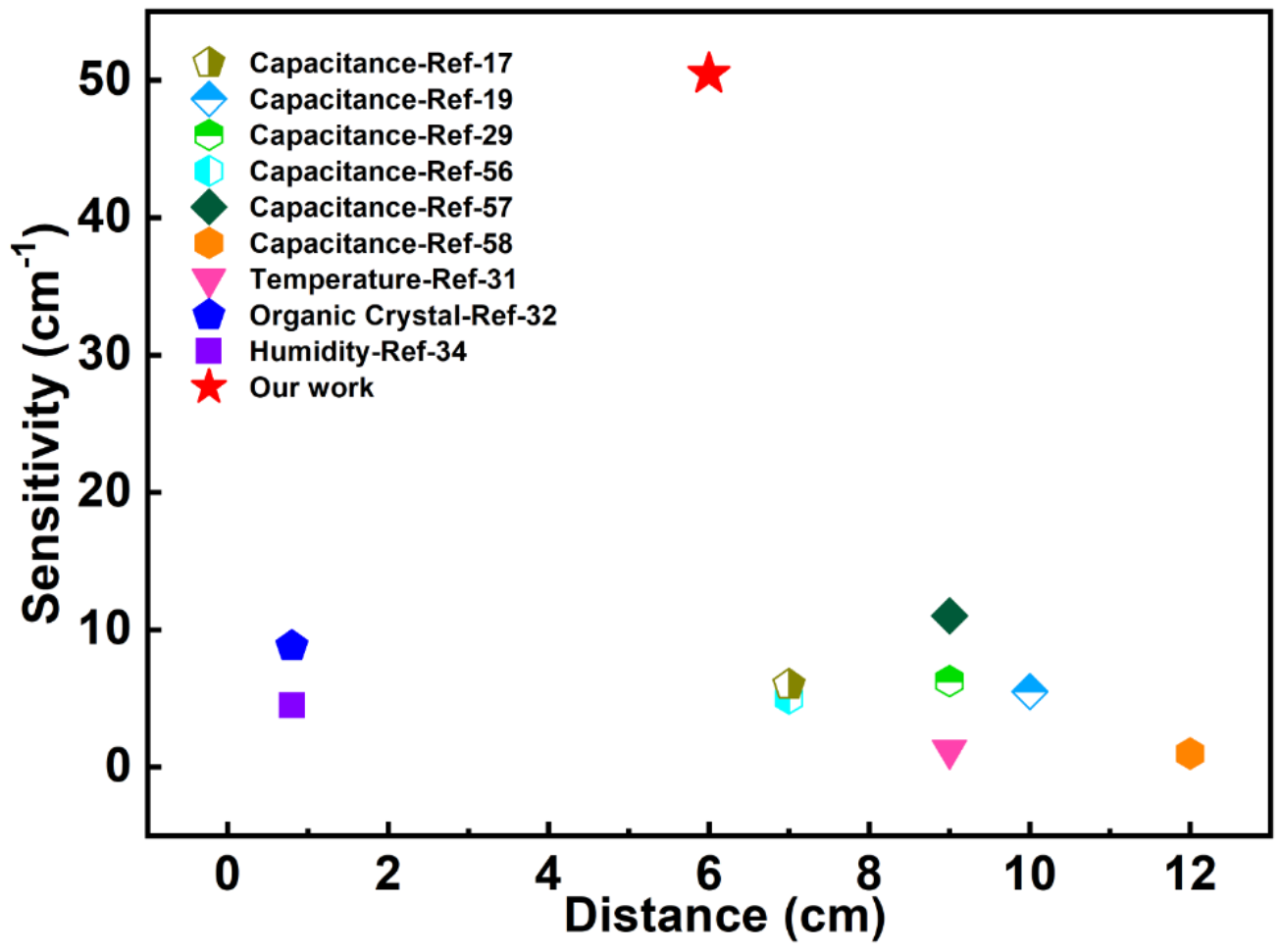

Figure S6. Comparison of the sensitivity and sensing distance of flexible proximity sensors based on different sensing mechanisms. 


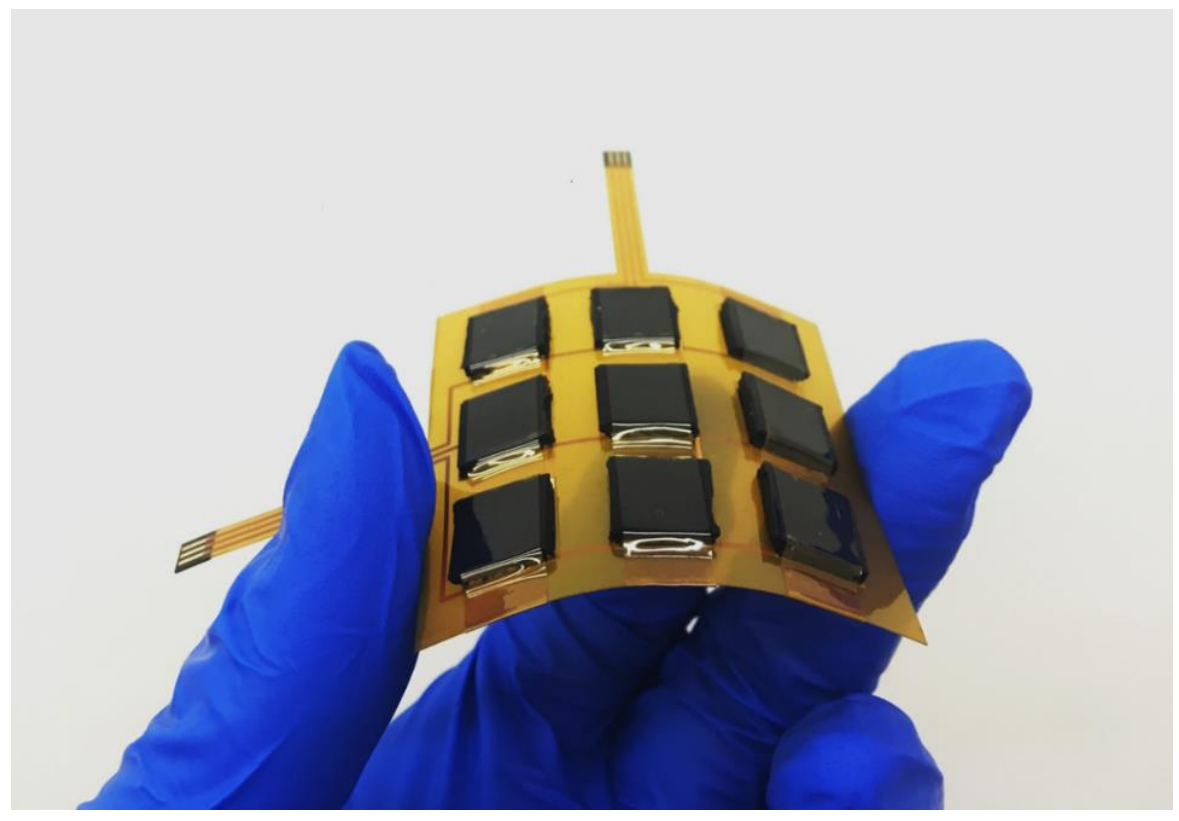

Figure S7. Optical image of the flexible magneto-piezoresistive proximity sensor array 